\title{
Primate Reaching Cued by Multichannel Spatiotemporal Cortical Microstimulation
}

\author{
N. A. Fitzsimmons, ${ }^{1,4}$ W. Drake, ${ }^{1,4}$ T. L. Hanson, ${ }^{1,4}$ M. A. Lebedev, ${ }^{1,4}$ and M. A. L. Nicolelis ${ }^{1,2,3,4}$ \\ Departments of ${ }^{1}$ Neurobiology, ${ }^{2}$ Biomedical Engineering, and ${ }^{3}$ Psychological and Brain Sciences and ${ }^{4}$ Center for Neuroengineering, Duke University, \\ Durham, North Carolina 27100
}

\begin{abstract}
Both humans and animals can discriminate signals delivered to sensory areas of their brains using electrical microstimulation. This opens the possibility of creating an artificial sensory channel that could be implemented in neuroprosthetic devices. Although microstimulation delivered through multiple implanted electrodes could be beneficial for this purpose, appropriate microstimulation protocols have not been developed. Here, we report a series of experiments in which owl monkeys performed reaching movements guided by spatiotemporal patterns of cortical microstimulation delivered to primary somatosensory cortex through chronically implanted multielectrode arrays. The monkeys learned to discriminate microstimulation patterns, and their ability to learn new patterns and new behavioral rules improved during several months of testing. Significantly, information was conveyed to the brain through the interplay of microstimulation patterns delivered to multiple electrodes and the temporal order in which these electrodes were stimulated. This suggests multichannel microstimulation as a viable means of sensorizing neural prostheses.
\end{abstract}

Key words: microstimulation; brain-machine interface; primate; somatosensory; discrimination; neuroprosthetics

\section{Introduction}

For more than a century, electrical stimulation has been used to probe brain circuitry and function in both humans (Penfield and Boldrey, 1937; Penfield and Rasmussen, 1950; Ransom, 1892) and animals (Fritsch and Hitzig, 1870; Ferrier, 1873, 1875). The effects of electrical stimulation depend on the location and parameters of stimulation. Stimulation of large brain areas typically hinders information processing (Pascual-Leone et al., 2000; Chambers and Mattingley, 2005; Kammer, 2006). Conversely, stimulation of small areas, termed microstimulation, can evoke motor and sensory effects that mimic the functional contribution of the stimulated area (Tehovnik, 1996; Graziano et al., 2002; Cohen and Newsome, 2004; DeAngelis and Newsome, 2004; Tehovnik et al., 2006). In the 1950s, neurobiologists began operantly conditioning animal behavior using electrical stimulation of the brain as a conditioned stimulus (Doty et al., 1956; Nielson et al., 1962; Doty, 1965, 1969) or a reinforcement (Olds and Milner, 1954). More recently, microstimulation of sensory areas has been shown to produce perceptual effects such as visual sensation (Bartlett et al., 2005), biasing the perception of visual

\footnotetext{
Received Dec. 7, 2006; revised April 5, 2007; accepted April 9, 2007.

This work was supported by the Defense Advanced Research Projects Agency, Defense Science Office, and the Space and Naval Warfare Systems Center, San Diego Contracts N66001-02-C-8022 and N66001-06-C-2019 (M.A.L.N.) and by National Institutes of Health Grant NS40543 (M.A.L.N.). We thank Dragan Dimitrov for performing the implantation surgery; Gary Lehew for designing the electrode implants; Aaron Sandler, Laura Oliveira, and Parag Patil for surgical assistance; lan Peikon for engineering and programming support; Steven Wise for reading the first draft of this manuscript and providing excellent suggestions; and Susan Halkiotis for manuscript preparation.

Correspondence should be addressed to M. A. L. Nicolelis, Department of Neurobiology, Bryan Research Building, Room 327E, Box 3209, Duke University Medical Center, 101 Research Drive, Durham, NC 27100. E-mail: nicoleli@neuro.duke.edu.

D0I:10.1523/JNEUROSC1.5297-06.2007

Copyright $\odot 2007$ Society for Neuroscience $\quad$ 0270-6474/07/275593-10\$15.00/0
}

motion (Salzman et al., 1990, 1992; Britten and van Wezel, 1998) or face recognition (Afraz et al., 2006), and mimicking the somatosensory perception of flutter (Romo et al., 1998, 2000; de Lafuente and Romo, 2005). Impressively, Talwar et al. (2002) used microstimulation of the rat barrel cortex to guide rats through a complex terrain. Although it is often difficult to prove that animals experience perceptions during microstimulation, microstimulation-induced perceptions have been demonstrated in humans (Penfield and Perot, 1963; Brindley and Lewin, 1968; Dobelle et al., 1976; Davis et al., 1998; Kiss et al., 2003; Ohara et al., 2004).

With the development of multielectrode implants (Nicolelis et al., 2003) and the concurrent advances of brain-machine interfaces (Chapin et al., 1999; Wessberg et al., 2000; Taylor et al., 2002; Carmena et al., 2003; Lebedev et al., 2005; Lebedev and Nicolelis, 2006), there is renewed interest in microstimulation as a means of providing the brain with an artificial sensory channel. Such a channel could recover sensation lost because of a neurological disorder or it could convey information from sensors of a prosthetic limb (Berger et al., 2005; Middlebrooks et al., 2005; Lebedev and Nicolelis, 2006; Wickelgren, 2006). Although this idea is intriguing, two critical issues must be addressed: (1) whether such artificial sensation can be improved by using multichannel microstimulation, and (2) whether microstimulation in this application is suitable for long-term usage. We explored the first issue by testing the capacity of owl monkeys to discriminate multichannel microstimulation of increasing complexity. We investigated the second issue by testing the long-term efficacy of microstimulation.

Two monkeys were previously trained in a reaching task in which target location was cued by vibrotactile stimuli (Sandler, 2005). Here, skin vibration was replaced by microstimulation of 


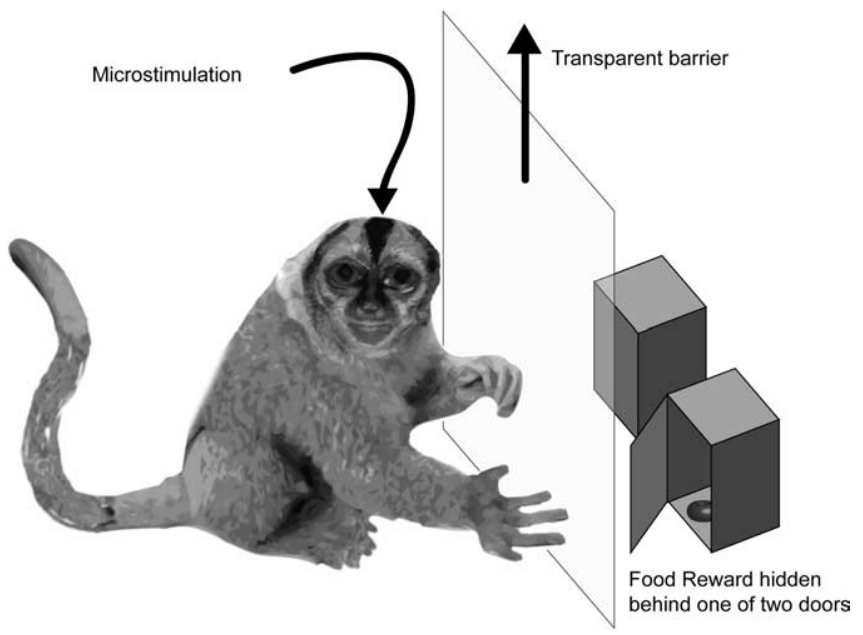

Figure 1. Experimental setup. Owl monkeys, while behind a transparent barrier, were cued to the location of a food reward through cortical microstimulation delivered to $S 1$ in the left hemisphere. After a $1 \mathrm{~s}$ delay following microstimulation, the barrier was raised, and the monkeys reached with the right arm to one of two doors. After opening the correct door, they retrieved a food reward, or alternatively after opening the incorrect door, they did not get any reward.

the primary somatosensory cortex (S1) delivered through multiple electrodes. During these long-term experiments, the monkeys' performance and their capability to learn new behavioral contingencies steadily improved.

\section{Materials and Methods}

Two adult female owl monkeys (Aotus trivirgatus) were implanted previously (Sandler, 2005) with microwire arrays in several cortical areas: primary somatosensory (S1), primary motor (M1), dorsal premotor, and posterior parietal cortices. These implants remained viable for electrophysiological recordings after more than four years. In the experiments reported here, we used S1 implants to deliver microstimulation patterns of different levels of complexity. S1 sites used for microstimulation corresponded to cutaneous receptive fields located on the monkeys' hands. In monkey 1 , two of the stimulation sites had clear receptive fields in the central palm of the right hand, whereas the remaining two sites corresponded to glabrous skin of the index and middle fingers of the same hand. In monkey 2, receptive fields of the stimulated sites were on the hand, although not as clearly defined as in monkey 1 . These receptive fields were different from the location of the vibratory cues, which were delivered to the shoulder. Stimulation parameters (current amplitude of $100-150 \mu \mathrm{A}$, pulse duration of $0.1 \mathrm{~ms}$ and a rate of pulse trains of $100 \mathrm{~Hz}$ ) were in the same range as that used previously for $\mathrm{S} 1$ microstimulation in rhesus monkeys (Romo et al., 1998, 2000; de Lafuente and Romo, 2005), as well as in other cortical microstimulation studies (Murasugi et al., 1993; Tehovnik et al., 2006).

Here, multichannel cortical microstimulation was used as a cue that guided reaching movements. Each monkey performed a reaching and grasping task that consisted of retrieving a food item from one of two locations covered by opaque doors (Fig. 1). Both monkeys were trained previously to guide their reaches by peripheral vibrotactile cues applied to their upper arms (Sandler, 2005). In this study, we used cortical microstimulation to cue the same behavior. Additionally, the initial time course (Fig. $2 \mathrm{~B}$ ) of the microstimulation pulses was the same as the time course for the vibratory stimulus that had been learned previously, except the microstimulation was delivered at $100 \mathrm{~Hz}$ instead of the $60 \mathrm{~Hz}$ used in vibratory stimulation.

The complexity of microstimulation patterns was progressively increased as the monkeys learned new discrimination tasks (Fig. 2). The patterns delivered to each electrode consisted of $100 \mathrm{~Hz}$ trains of electrical current pulses that were alternatively turned on and off, forming temporal patterns. Spatial patterns of microstimulation were formed by triggering microstimulation on different electrodes in different orders: "waves" of microstimulation were created, which propagated in different directions (Fig. 2E). Importantly, in all cases in which the monkeys discriminated between two microstimulation patterns, these patterns were never different in the total number of electrical pulses delivered through the stimulating electrodes. The difference was in the temporal and/or spatial patterns of microstimulation, not in the electrical charge passed through the electrodes.

Surgical and electrophysiological procedures. All surgical and behavioral procedures conformed to the National Research Council Guide for the Care and Use of Laboratory Animals and were approved by the Institutional Animal Care and Use Committee. During the implantation surgery (Kralik et al., 2001), each of the two adult female owl monkeys ( $A$. trivirgatus), weighing $800-850 \mathrm{~g}$, was anesthetized using $1.5-2.0 \%$ isoflourene and placed in a stereotaxic apparatus. A series of small craniotomies was made, both to grant access to the brain for the microwire arrays and for anchoring the dental acrylic to the skull. In each animal, multiple microwire arrays were chronically implanted in several cortical areas. The cortical areas implanted were primary somatosensory (S1), primary motor (M1), dorsal premotor (PMd), and posterior parietal (PP) cortices. Either $2 \times 8$ or $4 \times 8$ arrays of $50 \mu \mathrm{m}$ Teflon-coated steel, spaced $300 \mu \mathrm{m}$ apart, were used. The placement of the electrodes was accomplished using stereotaxic coordinates. Connectors for the arrays were embedded in a head cap made of dental acrylic.

Electrophysiological recordings and microstimulation. During the experimental sessions, the monkeys were placed in a custom-designed chair enclosure that restricted them to a sitting position while allowing for free arm motion and minimal head motion. Head stage amplifiers, or a custom microstimulation adapter were attached to the head-cap connectors. A flexible wire harness, in turn, connected the headstages to a 96-channel Plexon (Dallas, TX) recording system and the custom microstimulation adapter to the microstimulation device. The Plexon system was used for receptive field testing and, in concluding sessions, for obtaining neuronal recordings simultaneously with microstimulation.

We used several patterns of microstimulation. Initially, microstimulation was delivered to S1 through pairs of Iso-Flex stimulus isolators operating in constant current mode, with one isolator per pair producing the anodic current pulse and the other producing the cathodic current pulse. Triggering pulses were delivered to the isolators via the A.M.P.I. Master-8 programmable stimulator. The isolators were interfaced with the connectors mounted in the head caps via custom cabling. Later, this microstimulation hardware was replaced with a custom built, fourchannel, constant-current, biphasic, bipolar microstimulator controlled by custom software. The output voltage of each channel was supplied by two cascaded $1 \mathrm{~W}$ miniature isolated switching power supplies; these, in series, generated voltages up to $90 \mathrm{~V}$. This voltage was switched to the two bipolar electrodes through an optically isolated h-bridge in series with a simple current control circuit. The latter consisted of an optically isolated digital to analog converter (DAC), a current sense resistor, an opamp, and MOSFET (metal-oxide semiconductor field-effect) transistor in a feedback topology where the regulated current was set by the DAC output. Input to the DAC, like the h-bridge, was optically isolated; to conserve digital lines, the anodic and cathodic enable lines were switched via a global signal to control serial input to the 12-bit DAC register. The digital control lines for enabling the anodic and cathodic legs of the h-bridge were controlled directly by a National Instruments PCI-6533 card.

This stimulator was run continuously at a sampling rate of $100 \mathrm{kHz}$ via card-initiated direct memory access (DMA) through customized Linux device drivers. The DMA buffer was, in turn, serviced by a $\mathrm{C}++$ program with, effectively, up to four nested periods and duty cycles. These parameters were controlled via a Python script. The script permitted the user to control the stimulator from the Internet or programmatically via http GET commands. The Python script supported arbitrary loadable presets, which were stored in a MySQL database on the same Linux computer.

Stimulation parameters were similar to those used previously in S1 microstimulation in rhesus monkeys (Romo et al., 1998, 2000). These parameters were first tested by stimulating the motor cortex. We started with very low amplitude stimulation via electrodes in motor cortex, grad- 
A

Microstimulation Waveform
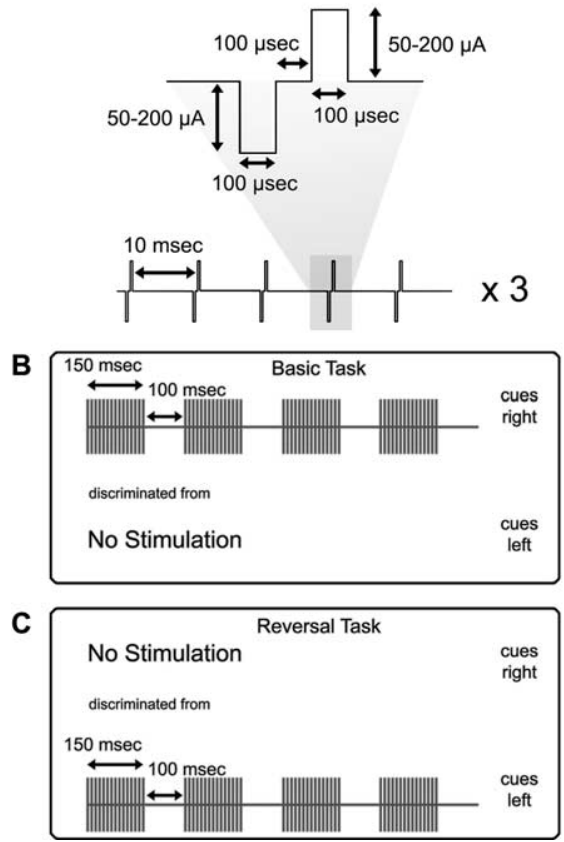

Figure 2. Temporal patterns of microstimulation. $\boldsymbol{A}$, In all sessions, stimulation pulses were delivered biphasically, with a cathodic phase preceding an anodic phase of equal amplitude. Pulse width, pulse delay, and frequency were kept constant at 0.1 $\mathrm{ms}, 0.1 \mathrm{~ms}$, and $100 \mathrm{~Hz}$, respectively. For all tasks except the one in which psychometric curves were measured, current amplitudes were held constant at 0.1 and $0.15 \mathrm{~mA}$ for monkeys 1 and 2, respectively. To construct the psychometric curves, we varied the stimulation currents between 0.05 and $0.2 \mathrm{~mA}$. $\boldsymbol{B}-\boldsymbol{E}$, Pulse bouts of $150 \mathrm{~ms}$ with $100 \mathrm{~ms}$ delays were used in the basic $(\boldsymbol{B})$, reversal (C), and spatiotemporal $(\boldsymbol{E})$ tasks while a second stimulation waveform consisting of $300 \mathrm{~ms}$ pulse bouts with $200 \mathrm{~ms}$ delays was also used in the temporal discrimination task (D). By keeping the low-level stimulation parameters constant between the two cues in the temporal and spatiotemporal tasks, the absolute charge injection was kept constant. In $\boldsymbol{E}$, electrode pairs (EP1-EP4) designate the sequential stimulus and ground electrode pairs along the linear electrode array.

ually raising the amplitude and frequency until a motor response was detected. Based on these estimations, we chose an initial stimulating current amplitude of $100-150 \mu \mathrm{A}$, pulse duration of $0.1 \mathrm{~ms}$, with an interpulse interval of $0.05-0.1 \mathrm{~ms}$ to avoid any interaction between the pulses because of imperfect stimulus decays of the two pulses making up the biphasic, and a rate of pulse trains of $100 \mathrm{~Hz}$. The pulse duration used in this study was the result of our empirical exploration to find the shortest pulse duration that worked, thus helping to avoid long-term tissue damage. Additionally, there were two issues that prevented the delivered current pulses from being ideal square pulses, both of which we compensated for. First, the components of the stimulation circuit (electrodes and custom wiring harness) added a capacitive load to the stimulator. Second, the optical components used the stimulation hardware had limited switching speed. In our setup, the switching limitations had a slightly larger effect than the capacitive terms, and the combination of these two created approximately exponential ramps, which reached $90 \%$ of their maximum in $\sim 30 \mu$ s. To compensate for the decrease of the delivered charge that resulted from these ramps, the duration of the command pulse was lengthened, making the net charge delivered equal to that which would have been delivered by a perfect rectangular pulse. The injected charge, which induces an electric field, is the parameter most related to the efficacy of stimulation. Because charge is a product of pulse width (or duration) and amplitude, both of these parameters influence the efficacy of microstimulation. In our analyses of psychometric curves, we modulated amplitude to modulate charge delivery, while keeping pulse width fixed.

Behavioral task. Two owl monkeys performed a reaching and grasping task. Each monkey sat comfortably in a primate chair. Two Plexiglas screens, one opaque, one transparent, separated the monkey from two small opaque doors, one to the right of the midline and one to the left. Food was hidden behind one of the doors, and the monkeys were behav- iorally conditioned to choose the location of the food by either peripheral vibrotactile cues or intracortical microstimulation cues (Fig. 1). After the food reward was hidden, the opaque screen was lifted and trials began with a $5 \mathrm{~s}$ period, during which the animal was behind the transparent Plexiglas screen and able to see the closed doors but unable to open them. During the first $4 \mathrm{~s}$, vibrotactile or microstimulation cues were delivered. Then, after a $1 \mathrm{~s}$ pause, the transparent screen was raised, and the monkey reached toward and opened one of the two doors. If the door selection was correct, the monkey obtained the food located behind the door. The monkey could make only one selection per reach, because the experimenter quickly took the food away if the monkey failed.

Trials occurred once every $30 \mathrm{~s}$ on average with $\sim 2$ s variability in the intertrial delay. Incorrect reaches were followed by repetition of the same type of trial. This design of trial presentation prevented the animals from becoming biased to a particular door but also made the trials that followed incorrect trials special, because the monkeys could use the previous failure as a cue of food location. These special correction trials were treated separately in the analyses of the behavioral data. Successful trials were followed by trials where food location was determined using a random number generator. The monkeys performed 100 trials during each daily session.

Each monkey was first overtrained to perform the reaching task, which was cued by vibrotactile stimuli applied to its upper arms (Sandler, 2005). If the right arm was vibrated, the animal had to reach toward the rightmost target, and if the left arm was vibrated, the correct direction of reach was to the left. Later, in a reversal phase of the experiment, each monkey also learned to reach to the target opposite to the stimulated arm. After 2 years of testing using arm vibration as the cue, the stimulus was switched to intracortical microstimulation. All other aspects of the task remained the same after the switch to microstimulation. Because the microstimulation would be delivered unilaterally, to the left primary somatosensory cortex only, in the months immediately preceding the switch from vibrostimulation to microstimulation, both monkeys were overtrained on unilateral vibrostimulation discrimination, reaching performance levels above $85 \%$ correct. The monkeys had to detect the presence or absence of vibration on the arm contralateral to the site that would be stimulated when the vibrostimulation was replaced with electrical microstimulation. In this way, the necessary relearning required of the stimulation cue switch was minimized.

Here, we report only the data obtained in the experiments in which microstimulation was used. The time course of the delivered pattern of microstimulation reproduced the previously used pattern of vibratory cues (Sandler, 2005). Microstimulation cues consisted of $100 \mathrm{~Hz}$ trains of electrical pulses that were alternatively turned on for $150 \mathrm{~ms}$ and turned off for $100 \mathrm{~ms}$, this pattern being repeated 16 times (i.e., for $4 \mathrm{~s}$ ). Microstimulation amplitude was set to $100 \mu \mathrm{A}$ for monkey 1 and to $150 \mu \mathrm{A}$ for monkey 2 , because these values corresponded to levels slightly below the average threshold for eliciting motor responses using microstimulation of M1. Electrical pulses were delivered simultaneously to three S1 electrodes in a linear arrangement with an interelectrode separation of 300 $\mu \mathrm{m}$ (Fig. 2). The stimulation electrodes shared a common global ground. In most sessions, the ground electrode located on the stimulation array away from the stimulation sites was connected to the stimulator ground. Alternatively, in some early sessions, the ground screws embedded in the dental acrylic implant were used. 
In the basic task (Fig. $2 \mathrm{~B}$ ), the monkeys discriminated the presence or absence of microstimulation. The correct response was to reach toward the target contralateral to the cortical site being stimulated (i.e., S1 in the left hemisphere) if the stimulation was turned on and to reach toward the other target if there was no stimulation. Electrical pulses were delivered simultaneously to three S1 electrodes in a linear arrangement with an interelectrode separation of $300 \mu \mathrm{m}$ (Fig. 3). These microstimulation patterns closely resembled vibrotactile patterns in which the monkeys were previously overtrained (Sandler, 2005): $100 \mathrm{~Hz}$ trains of electrical pulses were alternatively turned on for 150 $\mathrm{ms}$ and turned off for $100 \mathrm{~ms}$, this pattern being repeated 16 times (Fig. $2 \mathrm{~A}$ ). After the monkeys learned the basic task, this contingency was reversed: the monkey was required to reach toward the target ipsilateral to the site of stimulation (Fig. 2C).

Additionally, to study the psychometric relationship between amplitude and discrimination in the basic task, five different stimulus amplitudes were used, ranging from 0 to $200 \mu \mathrm{A}$ in logarithmic steps. For a given session, microstimulation amplitude was fixed at a randomly selected value without replacement.

After learning this task, the monkeys were required to discriminate between two temporal patterns of microstimulation (Fig. 2D). The amplitude, frequency, pulse width, and the number of pulses were held constant between the two different stimulation patterns. The difference was that one pattern was made of 150ms-long trains of pulses (pulse rate, $100 \mathrm{~Hz}$ ) separated by pauses of $100 \mathrm{~ms}$ (i.e., the same pattern as in the basic task), whereas the other was made of 300-ms-long trains separated by pauses of $200 \mathrm{~ms}$. The duration of each pattern of microstimulation was $4 \mathrm{~s}$. The first stimulation pattern previously used in the basic task (Fig. 2B) instructed the monkey to select the door contralateral to the stimulation site (the left hemisphere). The second pattern (Fig. 2D) instructed the monkey to select the door ipsilateral to the stimulation site. Stimulation was always delivered to $\mathrm{S} 1$ in the left hemisphere.

In the spatiotemporal task, the monkeys were required to discriminate between two spatiotemporal patterns of microstimulation. Again, the amplitude, frequency, pulse width, and number of pulses were held constant with pulse trains lasting for $150 \mathrm{~ms}$, separated by pauses of $100 \mathrm{~ms}$. However, whereas in previous tasks, all electrodes delivered the same pattern simultaneously; in this case, the electrodes were broken down into four electrode and ground pairs (EP1-EP4). To instruct the monkey to select the contralateral door, EP1 was stimulated for $150 \mathrm{~ms}$, then a 100 $\mathrm{ms}$ pause, then EP2 for $150 \mathrm{~ms}$, and so on, producing a wave of stimulation from EP1 to EP4. The four electrode-pair sequence (EP1 to EP4) was repeated four times for a total of $4 \mathrm{~s}$. To instruct the ipsilateral door choice, the direction of the microstimulation wave was reversed, going from EP4 to EP1. In both stimulation patterns, each individual electrode pair delivered the same current pulses, the only difference being the spatiotemporal order in which these electrode pairs were stimulated (Fig. 2E).

Analyses of behavioral data. Experimental trials during each session were split into two separate groups for analysis: random trials and correction trials. Correction trials (see above) were treated separately because the food location in these trials did not change from the previous trial. Thus, to obtain food in a correction trial, a monkey had to simply switch its direction of reach from the previous trial. These trials were used to prevent the monkeys from developing any biased strategies. Specifically, the monkeys had previously selected the same door repeatedly, getting a food reward half the time without paying attention to the cue. The correc- tion trials were analyzed separately, and this analysis confirmed that the monkeys indeed learned to switch the direction of reach. All other trials, called random trials, were characterized by food placement according to a computer-generated random sequence. These trials were analyzed to determine cue discrimination performance, because the only information regarding food location available to the monkeys came from the cue.

Discrimination performance was quantified as the proportion of random trials performed correctly ( $0-1$ range, with 0.5 corresponding to a chance level of performance). The performance over a series of sessions was fit to a linear regression model:

$$
y=m x+y_{\text {offset }},
$$

where $y$ is discrimination performance, $x$ is experimental session number, $m$ is the learning slope, and $y_{\text {offset }}$ is the initial performance. In addition, a sigmoidal model was used:

$$
y=\frac{y_{\text {scale }}}{1+e^{k\left(x-x_{\text {thresh }}\right)}}+y_{\text {offset }},
$$

where $y_{\text {offset }}$ describes the minimum of the sigmoid, $y_{\text {scale }}$ is the height of the sigmoid, $x_{\text {thresh }}$ is the threshold or inflection point of the sigmoid, and $k$ is a factor describing the slope of the sigmoid (larger $k$ values denote steeper slopes). For each fit, regression statistics were calculated to determine the goodness of the fit.

In addition to describing changes in performance in time, Equation 2 was used for constructing psychometric curves in which $x$ corresponded to microstimulation amplitude.

\section{Results}

\section{Control sessions and correction trials}

In control sessions, we showed that the monkeys did not use any strategy related to the reward schedule, experimenter interaction, 
or any other unseen source of information, to improve their performance. In these sessions, food was randomly placed exactly as in the basic task, but no cue was delivered to indicate food location. Mean performance on these random control trials was 0.48 and 0.47 in monkeys 1 and 2, respectively, which was not significantly different from chance ( $t$ test; $p>0.05)$.

Throughout all sessions, mean performance in correction trials, when food location was not altered following an incorrect trial, was 0.93 and 0.87 for monkeys 1 and 2, respectively. Performance in these trials was consistently significantly above chance values ( $t$ test; $p<0.001)$ in all task types. These trials were excluded from the analyses described below.

\section{Basic task}

In this task, the monkeys discriminated the presence of cortical microstimulation (Fig. $2 \mathrm{~B}$ ). Although both monkeys were overtrained in the reaching task cued by arm vibration, their initial performance in the basic task cued by similarly patterned microstimulation was rather poor. This was despite the fact that the week before the start of the microstimulation cueing sessions they performed the task in which vibration was used as a cue with a 0.84 average performance on random trials for both monkeys 1 and 2 .

In the initial sessions of the basic cortical microstimulation task, the behavioral performance was close to chance level. However, both monkeys exhibited steady improvement in performance over the course of 40 sessions, eventually reaching levels that were significantly above chance (Fig. 4$)(t$ test; $p<0.001)$. monkey 1's learning curve was well fit with a linear regression model (Eq. 1) with an initial performance (intercept) of 0.58 and a learning rate (slope) of 0.009 increase in performance per session $\left(r^{2}=0.75 ; p<0.001\right)$. monkey 2's learning curve was also well fit with a linear regression model (intercept of 0.46 ; learning rate of $\left.0.010 ; r^{2}=0.77 ; p<0.001\right)$. Note that monkey 1's performance surpassed the chance level during the very first session, whereas monkey 2 remained at approximately chance level for the first 18 experimental days. This initial phase with little perceptible learning was followed by a large, single jump of $0.15-$ 0.20 in performance. Significantly, once monkey 2 learned to use microstimulation cues in the basic task, it was able to rapidly learn more challenging discrimination tasks in the experiments that followed.

\section{Psychometric curve}

To study the range of microstimulation amplitudes that could be used to deliver reliable sensory input to the brain, we examined the performance of these monkeys on the well-learned basic task as we altered the amplitude of intracortical microstimulation. The relationship between current pulse amplitude and performance was well described by a sigmoidal psychometric curve (Eq. 2) for each monkey with goodness of fit $\left(r^{2}\right)$ of 0.93 and 0.76 for monkeys 1 and 2, respectively (Fig. 5). The respective sigmoidal fits were

$$
y=\frac{0.49}{1+e^{-0.06(x-72)}}+0.48
$$

and

$$
y=\frac{0.34}{1+e^{-0.04(x-60)}}+0.44
$$

\section{Basic Amplitude Discrimination Task}
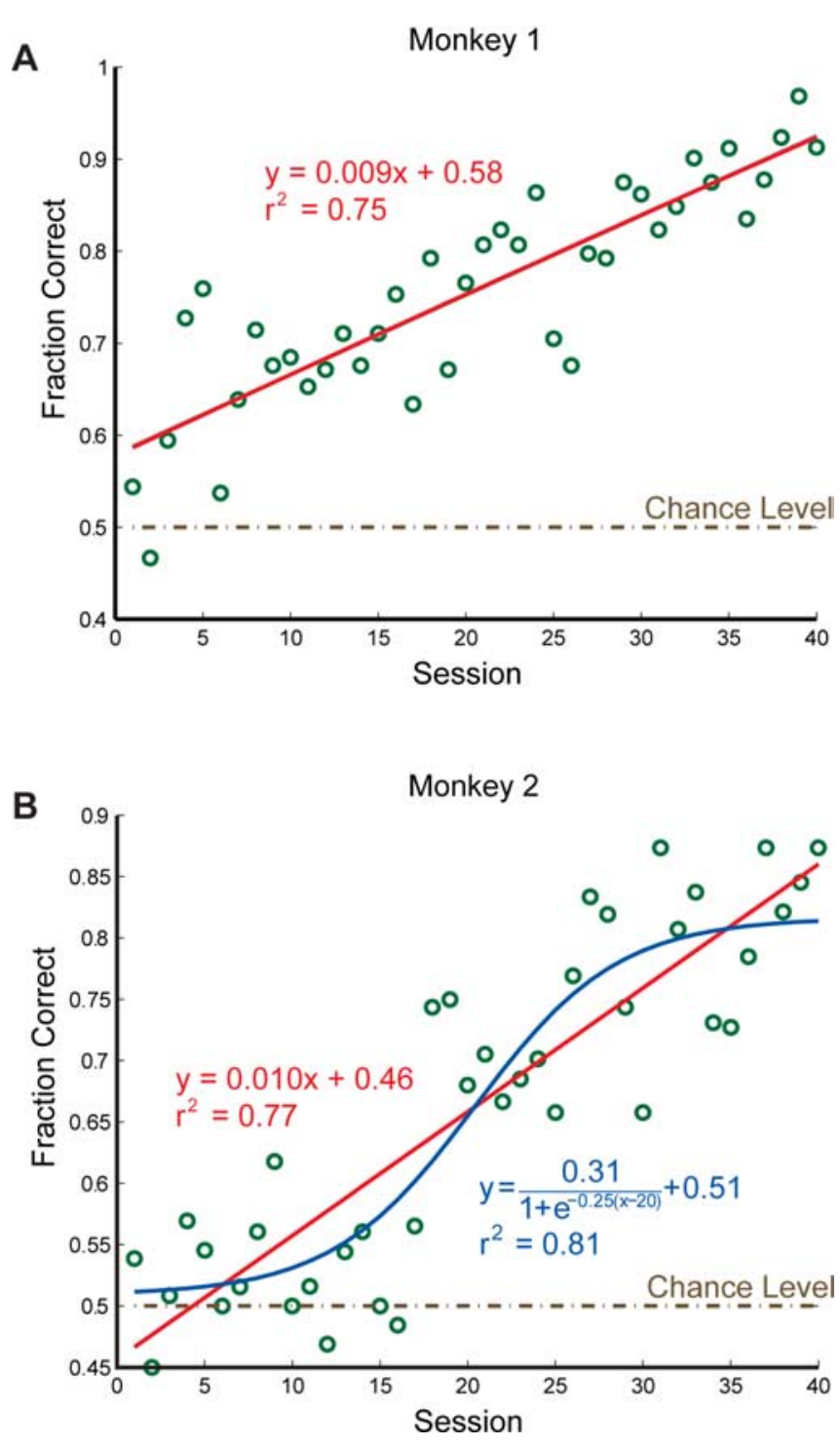

Figure 4. Improvement in discrimination performance (green) in the basic task in which monkey $1(\boldsymbol{A})$ and monkey $2(\boldsymbol{B})$ detected the presence of microstimulation and reached toward the target contralateral to the stimulated left hemisphere. Linear interpolation lines and their equations (red) are shown for monkeys 1 and 2, and for monkey 2, a sigmoidal interpolation line and its equation (blue) are shown. Chance performance level is 0.5 (brown).

The thresholds, defined here as the sigmoidal inflection point, were 72 and $60 \mu \mathrm{A}$ for monkeys 1 and 2, respectively, with $90 \%$ of maximum performance being achieved at 109 and $117 \mu \mathrm{A}$ (Fig. 5).

\section{Task reversal}

In a subsequent set of sessions, we investigated whether the monkeys could learn a new behavioral rule instructed by the same pattern of microstimulation (Fig. 2C). Although monkey 1 exhibited steady learning over the total course of the sessions, monkey 2's learning was characterized by a rapid improvement, followed by slower gradual enhancement of performance. Monkey 1 (intercept, 


\section{Psychometric Amplitude Analysis}
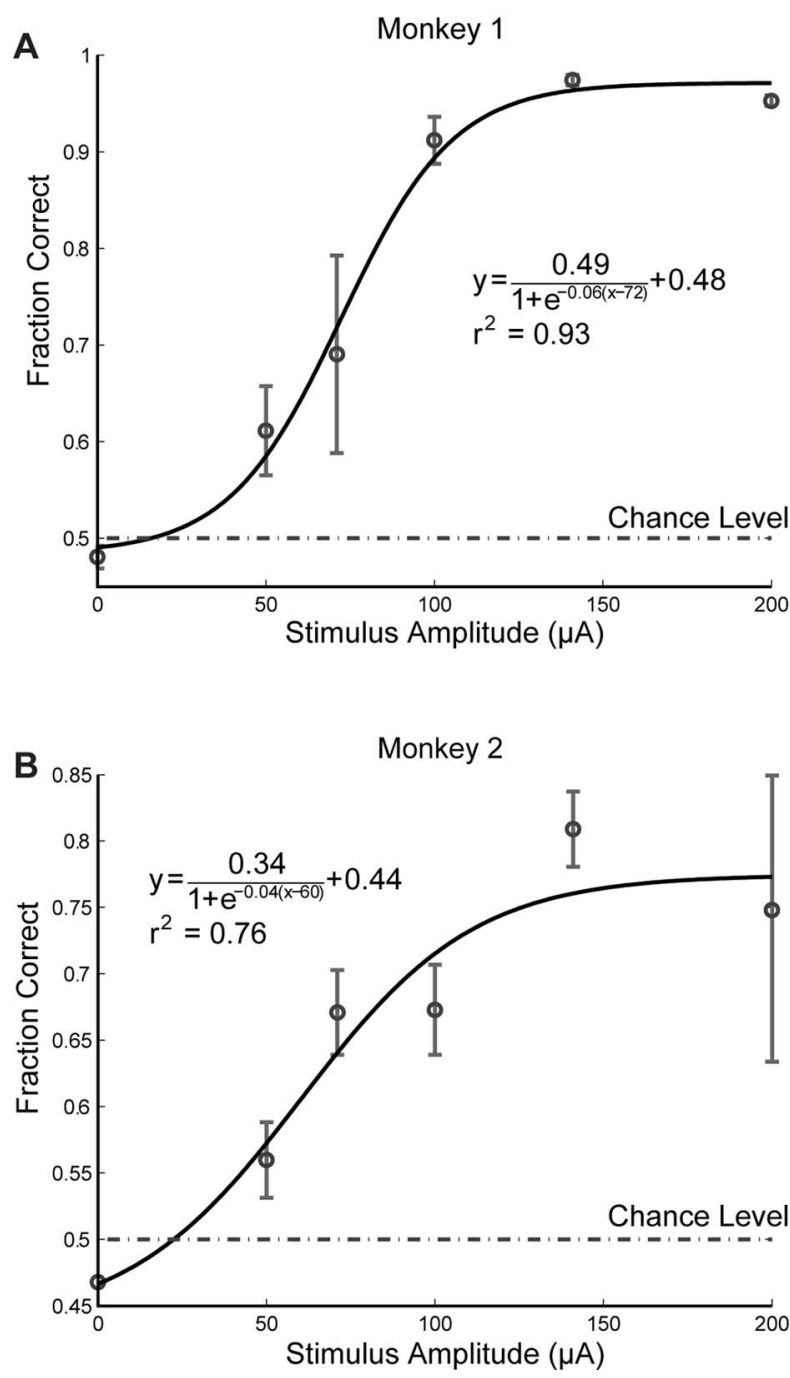

Figure 5. Psychometric curves for monkey $1(\boldsymbol{A})$ and monkey $2(\boldsymbol{B})$ characterizing the relationship between behavioral performance (green) quality and microstimulation amplitude. The curves were constructed by interpolating behavioral data with sigmoidal curves (blue). Chance performance level is 0.5 (brown).

0.29; slope, $0.064 ; r^{2}=0.97 ; p<0.001$ ) and monkey 2 (intercept, 0.67 ; slope, $\left.0.025 ; r^{2}=0.50 ; p<0.05\right)$ each exhibited linear learning curves (Fig. 6). The rapid jump in performance for monkey 2 is reflected in the higher intercept and lower slope. For both monkeys, high performance levels were reached over a much shorter time course compared with original learning of the basic task ( 0.064 and 0.025 slopes for reversal learning vs 0.008 and 0.010 for the original task for monkeys 1 and 2, respectively).

\section{Temporal pattern discrimination}

In the next set of experimental sessions (Fig. 2D), we investigated whether the same monkeys could discriminate the temporal characteristics of intracortical microstimulation, in addition to merely being able to detect the presence of stimulation. Learning for monkey 1 (intercept, 0.77 ; slope, $0.012 ; r^{2}=0.66 ; p<0.005$ ) and monkey 2 (intercept, 0.70 ; slope, $0.020 ; r^{2}=0.70 ; p<0.001$ ) proceeded steadily, with initial performance above chance but below final performance on the basic task (Fig. 7). Again, high performance levels were reached over a shorter time course than
Rule Reversal Task
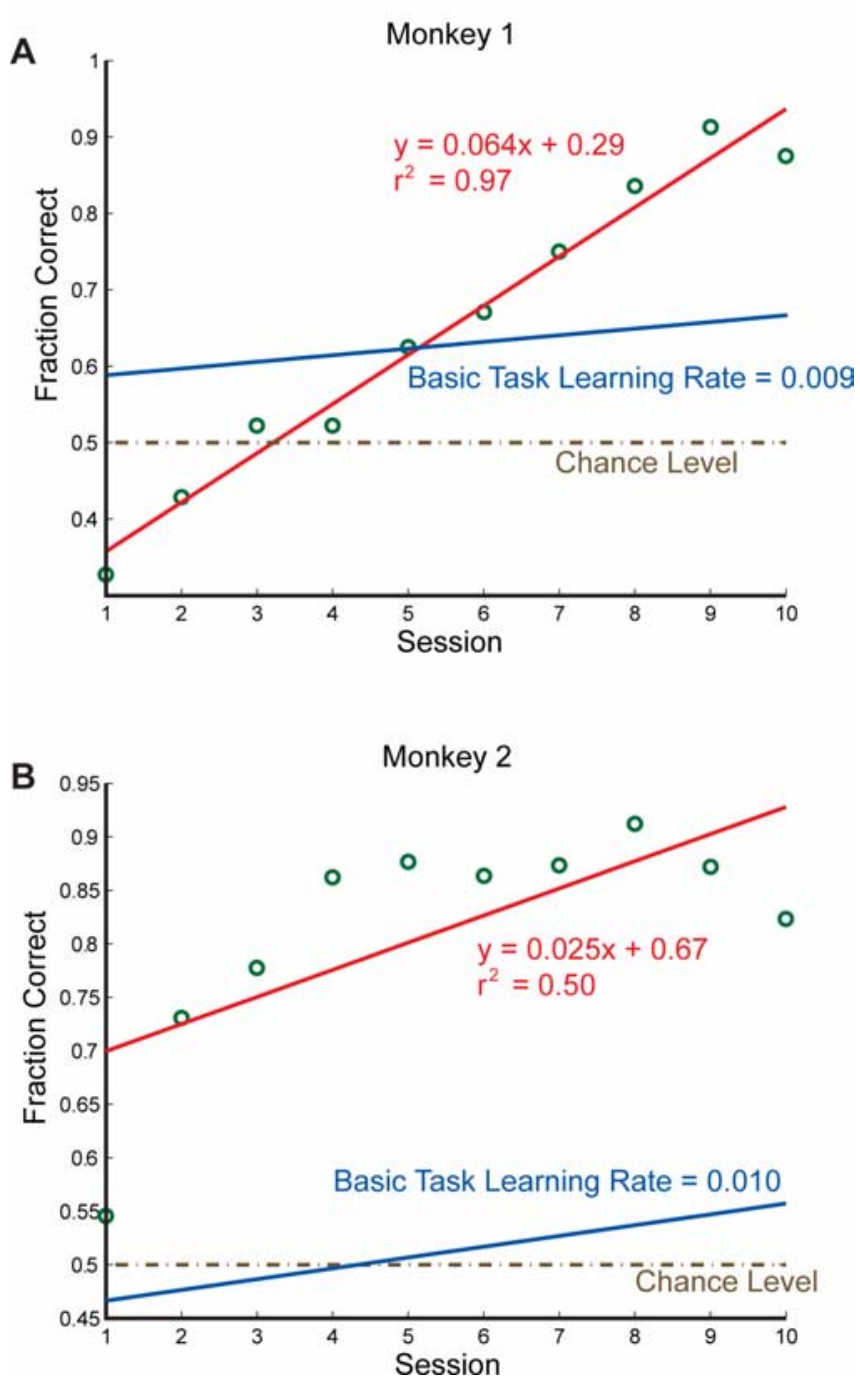

Figure 6. Improvement in discrimination performance (green) of reversal task [i.e., the task in which monkey $1(\boldsymbol{A})$ and monkey $2(\boldsymbol{B})$ were required to switch reach direction compared with the basic task]. Linear interpolation curves and their equations (red) are shown. For comparison, linear interpolation curves and learning rates (blue) for the basic task are shown as well. Chance performance level is 0.5 (brown).

that of initial learning in the basic task ( 0.85 performance level reached on the seventh and eighth sessions for temporal pattern learning vs the 32nd and 39th sessions for the original task for monkeys 1 and 2, respectively).

\section{Spatiotemporal pattern discrimination}

In the final set of experimental sessions (Fig. 2E), we investigated whether the owl monkeys could discriminate the spatiotemporal characteristics of intracortical microstimulation delivered through multiple implanted electrodes. Learning for monkey 1 (intercept, 0.85 ; slope, $0.011 ; r^{2}=0.36 ; p<0.001$ ) and monkey 2 (intercept, 0.77 ; slope, $0.020 ; r^{2}=0.65 ; p<0.001$ ) reached high values during the very first experimental session and proceeded steadily afterward. In this task, the learning reached a plateau of saturated performance after approximately the third or fourth sessions. Because of such quick saturation, a sigmoidal model- 
Temporal Pattern Discrimination Task
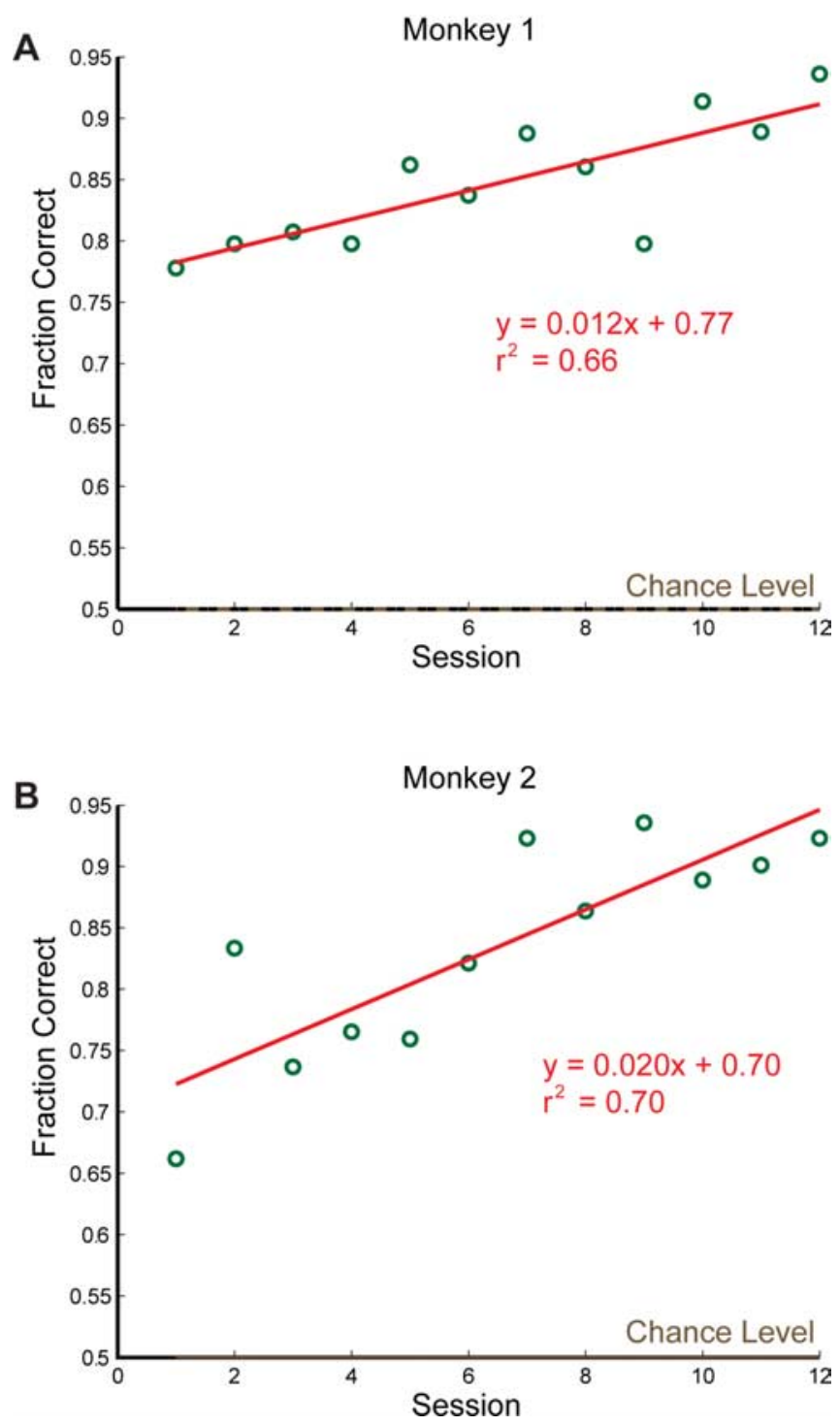

Figure 7. Improvement in performance (green) in the temporal discrimination task for monkey $1(\boldsymbol{A})$ and monkey $2(\boldsymbol{B})$. Linear interpolation curves and equations (red) are shown. Chance performance level is 0.5 (brown).

proved to be a better fit in both cases (Fig. 8). The respective sigmoidal fits for monkeys 1 and 2 were

$$
y=\frac{0.16}{1+e^{-2.74(x-2.06)}}+0.78\left(r^{2}=0.87\right)
$$

and

$$
y=\frac{106.12}{1+e^{-0.44(x+13.25)}}-105.18\left(r^{2}=0.76\right) .
$$

High performance levels were reached over a shorter time course than that of initial learning in the previous tasks ( 0.85 performance level reached on the second and third sessions for spatiotemporal pattern learning vs the 32nd and 39th sessions for the original task and the seventh and eighth sessions for temporal pattern learning for monkeys 1 and 2, respectively). In monkey 1 , there was significant intrasession learning during the first three

\section{Spatiotemporal Pattern Discrimination Task}
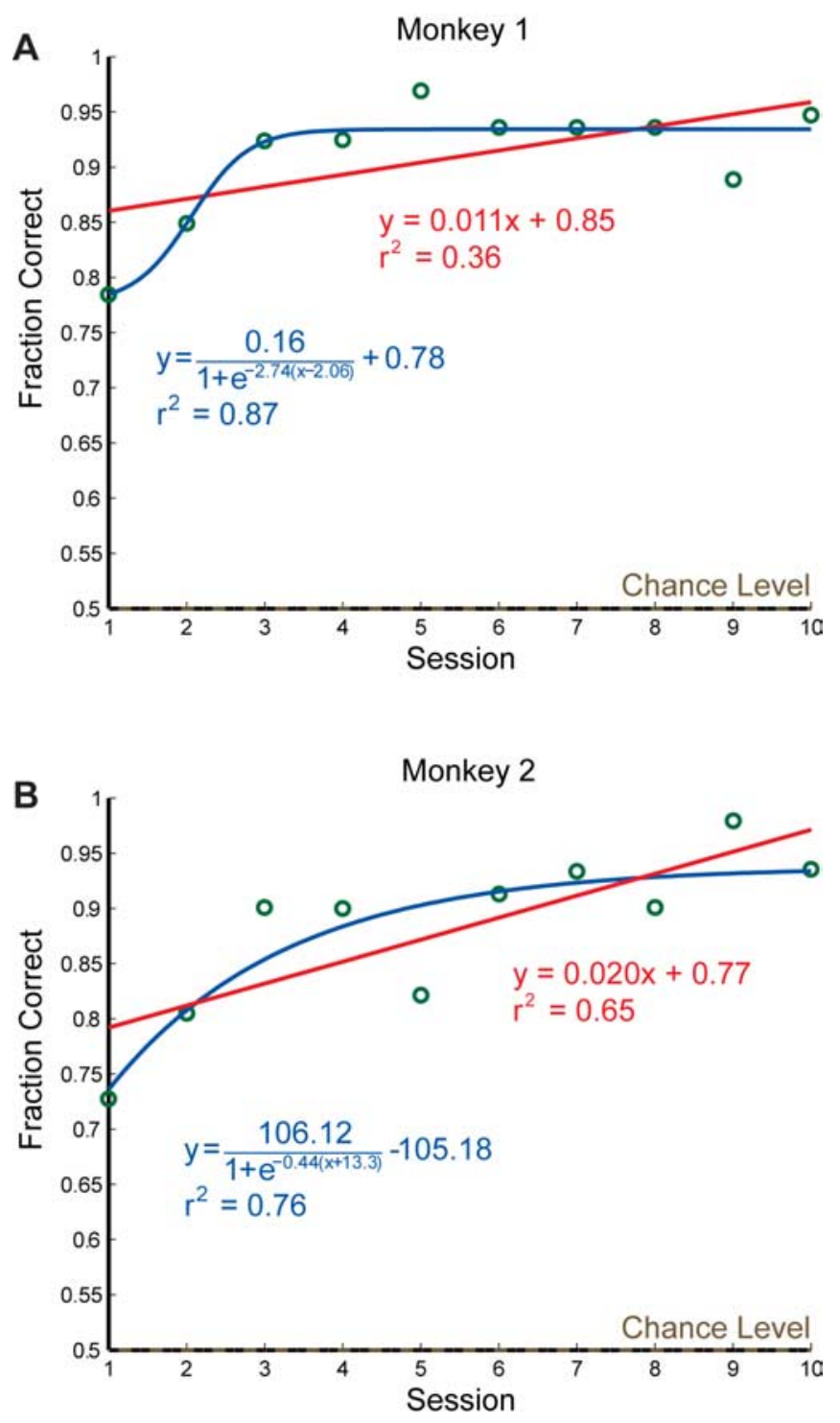

Figure 8. Improvement in performance (green) in the spatiotemporal discrimination task for monkey $1(\boldsymbol{A})$ and monkey $2(\boldsymbol{B})$. Linear (red) and sigmoidal (blue) interpolation curves and equations are shown. Chance performance level is 0.5 (brown).

sessions, with performance during the second half of each session significantly above the performance during the first half of the session $(p<0.05)$.

During several experimental sessions in which the monkeys discriminated spatiotemporal patterns of microstimulation, we also tested whether neuronal ensemble activity could be recorded while the microstimulation protocol was run (data not shown). The chronically implanted electrode arrays that were not being used for microstimulation were connected to a Plexon recording and spike discrimination system. Neuronal waveforms on nonstimulated arrays remained of the same quality as they were previously, before a microstimulation device being connected. Each microstimulation pulse produced an artifact of a distinct shape, the amplitude of which was approximately threefold or more higher compared with neuronal spikes. However, the custom stimulation hardware that is used minimizes the duration of each stimulus artifact, $\sim 1 \mathrm{~ms}$. During that $1 \mathrm{~ms}$, neuronal data cannot 
be recorded with our current technology, but when stimulating at $100 \mathrm{~Hz}$, the frequency used in these studies, only 10\% of the neuronal data is lost to the stimulation artifact. The remaining 90\% can be recorded normally and used to predict behavioral parameters. This technological achievement is important for further development of brain-machine interfaces with microstimulation feedback.

\section{Discussion}

In this study, we demonstrated that spatiotemporal patterns of microstimulation delivered to S1 through chronically implanted microelectrode arrays can convey useful information to the brain. Our experiments spanned several months. The monkeys progressed from learning to merely detect the presence of microstimulation to discriminating spatiotemporal patterns. The more they practiced, the easier it became for them to interpret novel microstimulation patterns. These results suggest that multichannel microstimulation can benefit neuroprosthetic applications that require sensory feedback.

\section{Methodological issues}

This study, along with previous ones from our laboratory (Wessberg et al., 2000; Sandler, 2005), showed certain benefits of using owl monkeys in the experiments that require long-term testing using chronically implanted microelectrodes. The first is the stability of the cortical implants. monkey 1 was implanted in 2003, and monkey 2 was implanted in 2001. Both monkeys retained good quality of recordings from their electrodes (Sandler, 2005), and their S1 electrodes remained effective for microstimulation. Long-term viability of neuronal recordings combined with the long-term efficiency of microstimulation makes owl monkeys a very useful species for developing neuroprosthetics for both extracting information from the brain and delivering information to the brain (Lebedev and Nicolelis, 2006).

Although our owl monkeys started learning the tasks cued by microstimulation relatively slowly, this characteristic may be actually used to the advantage of learning experiments: large amounts of neural and behavioral data can be collected over several recording sessions during which monkeys learn (Sandler, 2005). The stability of implanted electrodes assures the consistency of both neuronal recordings and microstimulation.

\section{Does microstimulation evoke perception?}

Numerous publications have raised the question of whether electrical microstimulation of sensory areas evokes perceptions (Romo et al., 1998, 2000, 2002; Liu and Newsome, 2000; Ohara et al., 2004; Otto et al., 2005; Tehovnik and Slocum, 2005). Romo et al. (1998), who studied single-channel acute microstimulation of $\mathrm{S} 1$ in rhesus monkeys, made a strong argument in favor of perceptions evoked by microstimulation being similar to those evoked by skin vibrotactile stimulation. Their rhesus monkeys had no difficulties comparing sensations evoked by vibrotactile stimulation to those evoked by S1 microstimulation and detecting the instances in which the frequency of microstimulation pulses matched the frequency of vibration.

The owl monkeys in our experiments initially did not perform as well as the rhesus monkeys in the study of Romo et al. (1998) after vibrotactile stimuli were replaced by microstimulation. This occurred despite the fact that both monkeys were previously overtrained in the reaching task cued by arm vibration. One possible explanation for the inability of owl monkeys to generalize the previously learned task to the microstimulation cue is that the owl monkeys may have experienced vibration-like sensations on their hands instead of their arms, where the mechanical vibrators had been placed previously. Another possibility is that microstimulation did not feel like vibration. In either case, the initially poor performance was probably related to the inability of owl monkeys to generalize the previously learned task to a somewhat different somatosensory cue.

The difference between the previously used skin vibration and the microstimulation cue, however, did not prevent the monkeys from learning the task under the novel conditions. In addition to learning the basic task, they learned the reversal, temporal, and spatiotemporal discrimination tasks. Significantly, learning in each new task occurred much more rapidly compared with the previous one. Perhaps with long-term use of microstimulation, the sensation that it evoked became more vivid. In support of this suggestion, use of a tactile stimulation as a channel for artificial vision was reported to allow subjects to develop qualitatively new perceptions (Bach-y-Rita, 1983; Segond et al., 2005).

\section{Does microstimulation disrupt neural processing?}

Although the monkeys in our study learned to discriminate the patterns of multichannel microstimulation, electrical stimulation can be disruptive for neural processing, preventing, or biasing normal behavior. Cortical functioning can be disrupted by both transcranial magnetic stimulation (Valero-Cabre et al., 2006) and microstimulation (Histed and Miller, 2006). We did not observe any deficits in motor performance or any bias to reach to a specific target after stimulation. We therefore conclude that disruption of neural processing, even if it affected the sensation from the monkeys' hands, did not have a role in the present task. Interference between continuous microstimulation (e.g., mimicking proprioception) and movements will have to be addressed by additional studies.

\section{Stimulation parameters}

Our psychometric measurements demonstrated that owl monkeys' ability to discriminate microstimulation patterns is similar to that previously reported in the studies of discrimination ability of rhesus monkeys using peripheral stimuli (LaMotte and Mountcastle, 1975) and cortical microstimulation (Murasugi et al., 1993; de Lafuente and Romo, 2005). As in previous studies, owl monkey performance for different stimulus amplitudes was well described by a sigmoidal curve. The psychometric curves that we and others obtained can be recommended generally for choosing microstimulation parameters in neuroprosthetics that attempt to create an artificial somatosensory channel.

\section{Long-term effects}

Running the microstimulation sessions for many days did not result in any neurological problems in the monkeys or deterioration in performance, which could be expected if microstimulation caused significant electrolytic damage to cortical tissue (Liu et al., 1999). On the contrary, with prolonged usage of microstimulation, performance steadily improved and eventually surpassed the levels previously observed for vibrotactile cues. The arrays in S1 used for stimulation were able to record multiunit activity throughout the study. The recording quality at stimulation sites remained at the initial level. Electrode arrays not used for stimulation remained viable for higher quality recordings and showed no observable changes after long-term stimulation on the implanted S1 arrays. Such long-term stability makes S1 microstimulation a sound candidate for creating an artificial somatosensory channel in neuroprosthetic applications. 


\section{Task reversal}

The ability of owl monkeys to reverse reach direction in the task cued by vibratory stimulation was demonstrated previously (Sandler, 2005). In this study, we showed that owl monkeys can do the same reversal when arm reach direction is cued by microstimulation. Such flexibility of behavioral response makes microstimulation a promising candidate for neurophysiological studies of rule representation and decision-making (Wise and Murray, 2000; Muhammad et al., 2006). Indeed, the delivery of microstimulation pulses can be controlled precisely and the subsequent neural responses measured in different brain areas. In combination with manipulations of behavioral rules imposed by the task, such an approach could be very productive for elucidating the neuronal computations between the stimulus arrival and the behavioral response. Furthermore, by using microstimulation at different neural processing stages, more can be learned about the specific layout of cortical circuitry and processing.

\section{Discrimination of temporal patterns}

Our demonstration that owl monkeys can learn to discriminate temporal characteristics of microstimulation is in agreement with the ability of rhesus monkeys to discriminate the frequency of S1 microstimulation (Romo et al., 2002). However, it is noteworthy that the stimuli that Romo et al. (2002) used contained different numbers of microstimulation pulses (i.e., different charges injected in the cortex), whereas in our experiments, the number of pulses was kept constant, which ensured that the monkeys based their discrimination on the temporal pattern of the stimulus rather than on its intensity.

\section{Discrimination of spatiotemporal patterns}

In this study, we demonstrated for the first time that owl monkeys can learn to discriminate spatiotemporal characteristics of cortical microstimulation created using multiple implanted electrodes. This suggests that there is depth and complexity to the animals' ability to "read out" microstimulation patterns. Adding the spatial dimension to the viable cortical microstimulation discrimination space significantly increases the potential throughput of information delivery to the brain. Indeed, by varying both temporal patterns of microstimulation and cortical sites to which it is delivered, as well as by taking advantage of stimulating selected cortical sites simultaneously, one can hope to significantly increase the amount of information conveyed to the brain in real time. Therefore, we suggest that multichannel microstimulation can be used in neuroprosthetic devices for delivery of complex, spatially, and temporally modulated signals to the brain. Information transfer rates that can be attained using microstimulation are of critical importance for neuroprosthetic application. Although our present estimation of the information capacity of multichannel microstimulation was limited by the modest learning ability of owl monkeys, our results indicate that the microstimulation "vocabulary" is extendable. Indeed, the monkeys learned new microstimulation patterns with progressively improving ease. Therefore, we expect that these monkeys would be able to learn a vocabulary of at least 10 different spatiotemporal stimuli, a number that almost certainly can be, by far, exceeded by human users of sensorized neural prostheses. The bandwidth of spatiotemporal patterns suitable for information delivery to the brain will have to be explored in future studies, both in temporal and spatial dimensions. In this study, we used relatively long, stereotypic, and repetitive microstimulation trains. It is possible that the very initial portions of these trains were sufficient for successful discrimination. In practical applications, information transfer rates could be increased by using much shorter microstimulation patterns with larger variability in their spatiotemporal structure.

\section{Encoding principles to be implemented}

To advance in the challenging tasks of replacing or providing new somatosensory sensations, we need a basic understanding of the responses that temporally patterned microstimulation produces in somatosensory cortical areas and the mechanisms by which the brain can use these responses to guide behavior. It would be naive to expect that neuronal activity patterns evoked by microstimulation would match normal somatosensory patterns. For instance, given the very complex excitatory and inhibitory effects produced by microstimulation (Butovas and Schwarz, 2003), it would be hard to reproduce rate-encoding features in S1 (Romo et al., 2002) using microstimulation. Increases in the frequency of microstimulation may not affect the total number of spikes firing in a predictable way, as excitatory periods begin to interfere with inhibitory periods. However, certain temporal patterns (Mountcastle et al., 1969, 1990; Lebedev et al., 1994) can be faithfully reproduced by cortical neurons. Indeed, during microstimulation, neuronal responses of cortical cells are clearly entrained to microstimulation pulses (Butovas and Schwarz, 2003), which provides a tool to deliver to the brain a variety of temporally patterned signals. Although the effect of microstimulation on average neuronal rates is somewhat unpredictable because of the aforementioned excitatory-inhibitory interactions (Butovas and Schwarz, 2003), it is more certain that groups of neurons in the stimulated area would entrain their activity to microstimulation patterns. Such synchronous, temporally patterned activity can then propagate to subsequent processing stages in the brain (Tehovnik et al., 2006). Although it would probably be impossible to replicate naturally occurring neural activity with current pulses emanating from metal electrodes inserted into the brain, the fidelity of transmission from electronic transducers could be sufficient for a variety of neuroprosthetic applications and thus enhance the quality of life for users of those devices in ways both measurable and immeasurable.

\section{References}

Afraz SR, Kiani R, Esteky H (2006) Microstimulation of inferotemporal cortex influences face categorization. Nature 442:692-695.

Bach-y-Rita P (1983) Tactile vision substitution: past and future. Int J Neurosci 19:29-36.

Bartlett JR, DeYoe EA, Doty RW, Lee BB, Lewine JD, Negrao N, Overman Jr WH (2005) Psychophysics of electrical stimulation of striate cortex in macaques. J Neurophysiol 94:3430-3442.

Berger TW, Ahuja A, Courellis SH, Deadwyler SA, Erinjippurath G, Gerhardt GA, Gholmieh G, Granacki JJ, Hampson R, Hsaio MC, LaCoss J, Marmarelis VZ, Nasiatka P, Srinivasan V, Song D, Tanquay AR, Wills J (2005) Restoring lost cognitive function. IEEE Eng Med Biol Mag $24: 30-44$.

Brindley GS, Lewin WS (1968) The sensations produced by electrical stimulation of the visual cortex. J Physiol (Lond) 196:479-493.

Britten KH, van Wezel RJ (1998) Electrical microstimulation of cortical area MST biases heading perception in monkeys. Nat Neurosci 1:59-63.

Butovas S, Schwarz C (2003) Spatiotemporal effects of microstimulation in rat neocortex: a parametric study using multielectrode recordings. J Neurophysiol 90:3024-3039.

Carmena JM, Lebedev MA, Crist RE, O’Doherty JE, Santucci DM, Dimitrov DF, Patil PG, Henriquez CS, Nicolelis MA (2003) Learning to control a brain-machine interface for reaching and grasping by primates. PLoS Biol 1:E42.

Chambers CD, Mattingley JB (2005) Neurodisruption of selective attention: insights and implications. Trends Cogn Sci 9:542-550.

Chapin JK, Moxon KA, Markowitz RS, Nicolelis MA (1999) Real-time con- 
trol of a robot arm using simultaneously recorded neurons in the motor cortex. Nat Neurosci 2:664-670.

Cohen MR, Newsome WT (2004) What electrical microstimulation has revealed about the neural basis of cognition. Curr Opin Neurobiol 14:169-177.

Davis KD, Kiss ZH, Luo L, Tasker RR, Lozano AM, Dostrovsky JO (1998) Phantom sensations generated by thalamic microstimulation. Nature 391:385-387.

de Lafuente V, Romo R (2005) Neuronal correlates of subjective sensory experience. Nat Neurosci 8:1698-1703.

DeAngelis GC, Newsome WT (2004) Perceptual "read-out" of conjoined direction and disparity maps in extrastriate area MT. PLoS Biol 2:E77.

Dobelle WH, Mladejovsky MG, Evans JR, Roberts TS, Girvin JP (1976) "Braille" reading by a blind volunteer by visual cortex stimulation. Nature 259:111-112.

Doty RW (1965) Conditioned reflexes elicited by electrical stimulation of the brain in macaques. J Neurophysiol 28:623-640.

Doty RW (1969) Electrical stimulation of the brain in behavioral context. Annu Rev Psychol 20:289-320.

Doty RW, Larsen RM, Ruthledge Jr LT (1956) Conditioned reflexes established to electrical stimulation of cat cerebral cortex. J Neurophysiol 19:401-415.

Ferrier D (1873) Experimental research in cerebral physiology and pathology. West Riding Lunatic Asylum Medical Reports 3:30-96.

Ferrier D (1875) The croonian lecture: experiments on the brain of monkeys (second series). Philos Trans R Soc Lond B Biol Sci 165:433-488.

Fritsch G, Hitzig E (1870) Über die elektrische Erregbarkeit des Grosshirns. Arch Anat Physiol Med Wiss 300-332.

Graziano MS, Taylor CS, Moore T (2002) Complex movements evoked by microstimulation of precentral cortex. Neuron 34:841-851.

Histed MH, Miller EK (2006) Microstimulation of frontal cortex can reorder a remembered spatial sequence. PLoS Biol 4:e134.

Kammer T (2007) Masking visual stimuli by transcranial magnetic stimulation. Psychol Res, in press.

Kiss ZH, Anderson T, Hansen T, Kirstein D, Suchowersky O, Hu B (2003) Neural substrates of microstimulation-evoked tingling: a chronaxie study in human somatosensory thalamus. Eur J Neurosci 18:728-732.

Kralik JD, Dimitrov DF, Krupa DJ, Katz DB, Cohen D, Nicolelis MA (2001) Techniques for long-term multisite neuronal ensemble recordings in behaving animals. Methods 25:121-150.

LaMotte RH, Mountcastle VB (1975) Capacities of humans and monkeys to discriminate vibratory stimuli of different frequency and amplitude: a correlation between neural events and psychological measurements. J Neurophysiol 38:539-559.

Lebedev MA, Nicolelis MA (2006) Brain-machine interfaces: past, present and future. Trends Neurosci 29:536-546.

Lebedev MA, Denton JM, Nelson RJ (1994) Vibration-entrained and premovement activity in monkey primary somatosensory cortex. J Neurophysiol 72:1654-1673.

Lebedev MA, Carmena JM, O’Doherty JE, Zacksenhouse M, Henriquez CS, Principe JC, Nicolelis MA (2005) Cortical ensemble adaptation to represent velocity of an artificial actuator controlled by a brain-machine interface. J Neurosci 25:4681-4693.

Liu J, Newsome WT (2000) Somatosensation: touching the mind's fingers. Curr Biol 10:R598-R600.

Liu X, McCreery DB, Carter RR, Bullara LA, Yuen TG, Agnew WF (1999) Stability of the interface between neural tissue and chronically implanted intracortical microelectrodes. IEEE Trans Rehabil Eng 7:315-326.

Middlebrooks JC, Bierer JA, Snyder RL (2005) Cochlear implants: the view from the brain. Curr Opin Neurobiol 15:488-493.

Mountcastle VB, Talbot WH, Sakata H, Hyvarinen J (1969) Cortical neuronal mechanisms in flutter-vibration studied in unanesthetized monkeys. Neuronal periodicity and frequency discrimination. J Neurophysiol 32:452-484.

Mountcastle VB, Steinmetz MA, Romo R (1990) Frequency discrimination in the sense of flutter: psychophysical measurements correlated with postcentral events in behaving monkeys. J Neurosci 10:3032-3044.

Muhammad R, Wallis JD, Miller EK (2006) A comparison of abstract rules in the prefrontal cortex, premotor cortex, inferior temporal cortex, and striatum. J Cogn Neurosci 18:974-989.
Murasugi CM, Salzman CD, Newsome WT (1993) Microstimulation in visual area MT: effects of varying pulse amplitude and frequency. J Neurosci 13:1719-1729.

Nicolelis MA, Dimitrov D, Carmena JM, Crist R, Lehew G, Kralik JD, Wise SP (2003) Chronic, multisite, multielectrode recordings in macaque monkeys. Proc Natl Acad Sci USA 100:11041-11046.

Nielson HC, Knight JM, Porter PB (1962) Subcortical conditioning, generalization, and transfer. J Comp Physiol Psychol 55:168-173.

Ohara S, Weiss N, Lenz FA (2004) Microstimulation in the region of the human thalamic principal somatic sensory nucleus evokes sensations like those of mechanical stimulation and movement. J Neurophysiol 91:736-745.

Olds J, Milner P (1954) Positive reinforcement produced by electrical stimulation of septal area and other regions of rat brain. J Comp Physiol Psychol 47:419-427.

Otto KJ, Rousche PJ, Kipke DR (2005) Microstimulation in auditory cortex provides a substrate for detailed behaviors. Hear Res 210:112-117.

Pascual-Leone A, Walsh V, Rothwell J (2000) Transcranial magnetic stimulation in cognitive neuroscience-virtual lesion, chronometry, and functional connectivity. Curr Opin Neurobiol 10:232-237.

Penfield W, Boldrey E (1937) Somatic motor and sensory representation in the cerebral cortex of man as studied by electrical stimulation. Brain 60:389-443.

Penfield W, Perot P (1963) The brain's record of auditory and visual experience: a final summary and discussion. Brain 86:595-696.

Penfield W, Rasmussen T (1950) The cerebral cortex of man: a clinical study of localisation of function. New York: Macmillan.

Ransom W (1892) A case illustrating kinaesthesis. Brain 15:437-442.

Romo R, Hernandez A, Zainos A, Salinas E (1998) Somatosensory discrimination based on cortical microstimulation. Nature 392:387-390.

Romo R, Hernandez A, Zainos A, Brody CD, Lemus L (2000) Sensing without touching: psychophysical performance based on cortical microstimulation. Neuron 26:273-278.

Romo R, Hernandez A, Salinas E, Brody CD, Zainos A, Lemus L, de Lafuente V, Luna R (2002) From sensation to action. Behav Brain Res 135:105-118.

Salzman CD, Britten KH, Newsome WT (1990) Cortical microstimulation influences perceptual judgements of motion direction. Nature 346:174-177.

Salzman CD, Murasugi CM, Britten KH, Newsome WT (1992) Microstimulation in visual area MT: effects on direction discrimination performance. J Neurosci 12:2331-2355.

Sandler AJ (2005) Neuronal correlates of somatosensorimotor learning and decision making in primates. $\mathrm{PhD}$ thesis, Duke University.

Segond H, Weiss D, Sampaio E (2005) Human spatial navigation via a visuo-tactile sensory substitution system. Perception 34:1231-1249.

Talwar SK, Xu S, Hawley ES, Weiss SA, Moxon KA, Chapin JK (2002) Rat navigation guided by remote control. Nature 417:37-38.

Taylor DM, Tillery SI, Schwartz AB (2002) Direct cortical control of 3D neuroprosthetic devices. Science 296:1829-1832.

Tehovnik EJ (1996) Electrical stimulation of neural tissue to evoke behavioral responses. J Neurosci Methods 65:1-17.

Tehovnik EJ, Slocum WM (2005) Microstimulation of V1 affects the detection of visual targets: manipulation of target contrast. Exp Brain Res 165:305-314.

Tehovnik EJ, Tolias AS, Sultan F, Slocum WM, Logothetis NK (2006) Direct and indirect activation of cortical neurons by electrical microstimulation. J Neurophysiol 96:512-521.

Valero-Cabre A, Rushmore RJ, Payne BR (2006) Low frequency transcranial magnetic stimulation on the posterior parietal cortex induces visuotopically specific neglect-like syndrome. Exp Brain Res 172:14-21.

Wessberg J, Stambaugh CR, Kralik JD, Beck PD, Laubach M, Chapin JK, Kim J, Biggs SJ, Srinivasan MA, Nicolelis MA (2000) Real-time prediction of hand trajectory by ensembles of cortical neurons in primates. Nature 408:361-365.

Wickelgren I (2006) Biomedical engineering. A vision for the blind. Science 312:1124-1126.

Wise SP, Murray EA (2000) Arbitrary associations between antecedents and actions. Trends Neurosci 23:271-276. 